\title{
The Effects of Body Mass Index on the Use of Patient-Controlled Intravenous Analgesia After Open Gastrointestinal Tumor Surgery: A Retrospective Analysis
}

This article was published in the following Dove Press journal:

Journal of Pain Research

\author{
Ting-Ting $\mathrm{Li}^{\mathrm{l}, *}$ \\ Liu-Lin Xiong ${ }^{2, *}$ \\ Jin Huang ${ }^{3}$ \\ Song Wen ${ }^{4}$ \\ Yan-Jun Chen' \\ Ting-Hua Wang' \\ Fei Liu (D) ${ }^{\prime}$
}

'Department of Anesthesiology, Institute of Neurological Disease, West China Hospital, Sichuan University, Chengdu, 610044, People's Republic of China; ${ }^{2}$ School of Pharmacy and Medical Sciences, Faculty of Health Sciences, University of South Australia, Adelaide, SA 5000, Australia; ${ }^{3}$ Laboratory Zoology Department, Institute of Neuroscience, Kunming Medical University, Kunming, People's Republic of China; ${ }^{4}$ Department of Pain, Affiliated Hospital of Zunyi Medical University, Guizhou, People's Republic of China

*These authors contributed equally to this work

Correspondence: Ting-Hua Wang Institute of Neurological Disease, Department of Anesthesiology, Translational Neuroscience Center, West China Hospital, Sichuan University, Chengdu 61004I,

People's Republic of China

Tel +86 87I-65939|80

Fax +86 87I-65922935

Email Wangth_email@I63.com

Fei Liu

Department of Anesthesiology, West China Hospital, Sichuan University, Chengdu 61004I, People's Republic of China

Tel +86 I8980605856

$\mathrm{Fax}+86$ 87I-85423593

Email 30333870@qq.com
Purpose: To investigate the impact of body mass index (BMI) on the analgesic effects and adverse reactions of patient-controlled intravenous analgesia (PCIA).

Methods: From 2017 to 2018, 390 patients undergoing open gastrointestinal surgery were reviewed at West China Hospital, Sichuan University. All used PCIA of sufentanil combined with dexmedetomidine and flurbiprofen axetil. According to their BMIs, they were placed into six groups: group A (BMI $\left.<18.5 \mathrm{~kg} / \mathrm{m}^{2}, 29\right)$, group B $\left(18.5 \mathrm{~kg} / \mathrm{m}^{2} \leq \mathrm{BMI}<22 \mathrm{~kg} / \mathrm{m}^{2}\right.$, 124), group C $\left(22 \mathrm{~kg} / \mathrm{m}^{2} \leq \mathrm{BMI}<24 \mathrm{~kg} / \mathrm{m}^{2}, 99\right)$, group D $\left(24 \mathrm{~kg} / \mathrm{m}^{2} \leq \mathrm{BMI}<26 \mathrm{~kg} / \mathrm{m}^{2}, 69\right)$, group E $\left(26 \mathrm{~kg} / \mathrm{m}^{2} \leq \mathrm{BMI}<28 \mathrm{~kg} / \mathrm{m}^{2}, 46\right)$ and group $\mathrm{F}\left(\mathrm{BMI} \geq 28 \mathrm{~kg} / \mathrm{m}^{2}, 23\right)$. Main data of the perioperative use of analgesics, postoperative visual analogue score (VAS), and adverse reactions were collected.

Results: Twenty-four hours (h) after surgery, patients in group A had a higher resting VAS than the other groups, especially $\mathrm{B}\left(p_{A-B}=0.011\right)$. VAS of patients during activity in group B was lower than those in group C $48 \mathrm{~h}$ after surgery $(p=0.013)$. Compared with groups B to $\mathrm{F}$, group A had a significantly lower incidence of hypertension ( $p=$ 0.012 ) and a significantly higher incidence of vomiting $24 \mathrm{~h}$ after surgery $(p=0.009)$. Binary logistic analysis found that higher age was a risk factor for vomiting $24 \mathrm{~h}$ after surgery (OR 1.158, $p=0.045$ ).

Conclusion: Using the same PCIA, patients with BMIs of less than $18.5 \mathrm{~kg} / \mathrm{m}^{2}$ had worse analgesia on the first day after surgery and were more likely to vomit. Postoperative analgesia and related experiences in patients with BMIs of less than $18.5 \mathrm{~kg} / \mathrm{m}^{2}$ need to be improved.

Keywords: postoperative nausea and vomiting, PONV, postoperative pain, sufentanil, dexmedetomidine, flurbiprofen axetil

\section{Introduction}

Postoperative patient-controlled intravenous analgesia (PCIA) is one of the most common methods in clinical practice, but the current PCIA cannot achieve a satisfactory clinical effect. ${ }^{1}$ Although patient-controlled epidural analgesia (PCEA) is more effective, ${ }^{2}$ it is associated with an increased risk of postoperative nausea and vomiting (PONV), ${ }^{3}$ hypotension, and complicated operations. ${ }^{4,5}$ Therefore, a new drug combination formulated for PCIA was designed at West China Hospital. It was prepared in combination with sufentanil, dexmedetomidine and flurbiprofen ester. Sufentanil is widely used in 
perioperative analgesia due to its strong analgesic effect and small influence on perioperative haemodynamics. ${ }^{6}$ However, with increase of analgesic dose, opioids are more likely to cause respiratory depression, gastrointestinal discomfort and drug resistance. Multi-mode analgesia with different modes of action may be beneficial to reduce the occurrence of adverse reactions. ${ }^{7}$ Dexmedetomidine is a highly selective $\alpha 2$ adrenal receptor agonist offering unique sedative and analgesic effects without respiratory inhibition. ${ }^{8}$ Some studies show that dexmedetomidine combined with sufentanil for PCIA could reduce postoperative pain scores and sufentanil consumption,9,10 but with a risk of bradycardia and hypotension. ${ }^{11}$ A review has shown that non-steroidal anti-inflammatory drugs have some analgesic effect without increasing adverse reactions in patients. $^{12}$ Through meta-analysis, Sun et al indicated that flurbiprofen can significantly reduce the incidence of postoperative nausea and vomiting (PONV). ${ }^{13}$ Moreover, previous studies showed that the new PCIA had better analgesia and less PONV than the old PCIA (sufentanil combined with tramadol). Nonetheless, some patients still needed additional analgesics to achieve satisfactory pain relief. And by regression analysis of the above analgesia regimen, we found that BMI was a risk factor for postoperative pain. ${ }^{14}$ Through a randomized-controlled study, Majchrzak et al found that obese patients (mean BMI of $34.1 \pm 3.2 \mathrm{~kg} / \mathrm{m}^{2}$ ) with lung cancer suffered more pain after surgery than non-obese patients (mean BMI of $24.9 \pm 3 \mathrm{~kg} / \mathrm{m}^{2}$ ), and that obese had longer durations of severe pain. ${ }^{15}$ Using a similar approach, Tashani et al demonstrated that the obese (BMI $\geqq 30 \mathrm{~kg} / \mathrm{m}^{2}$ ) were more sensitive to tenderness than those in a normal BMI range. ${ }^{16}$ In addition, one study showed that low BMI was an independent risk factor for colonoscopy pain. ${ }^{17}$ These studies suggest that patients with different BMIs had varying sensitivity to pain. However, there has been no research on the consequences of BMI on the analgesic effect and adverse reactions of PCIA. Thus, we studied 390 patients who used the same PCIA after surgery to understand the impact of BMI on PCIA.

\section{Materials and Methods}

\section{Grouping and Patients}

A total of 390 adult patients with open gastrointestinal tumors were reviewed at West China Hospital from October 2017 to July 2018. All patients received intravenous - inhalation combined anaesthesia, selective operation, and the same PCIA scheme, and no nerve block was used. Inclusion criteria: 1) Patients older than 18 years and younger than 80; 2) American Society of Anesthesiologists (ASA) level 1-2; 3) Elective gastrointestinal surgery. Exclusion criteria: 1) Anxiety or depression; 2) Preoperative Huaxi Mood Index (HEI) [Supplementary Material Page 1] greater than 12 (excluding patients with recent mood abnormalities); 3) Preoperative complicated with heart disease, severe anaemia, severe hypoproteinemia, hypertension grade 3 and diabetes with other diseases (only patients with grade 1-2 hypertension or diabetes or no complications were included); 5) Patients who did not use medium long-acting analgesics (tramadol, flurbiprofen, dezocine, tramadol) or more than two kinds of analgesics during the operation; 6) Patients with multiple tumor sites found intraoperatively; 7) Transferred to intensive care unit (ICU) after surgery; 8) PONV caused by the influence of gastric tube; 9) Postoperative pathological analysis showed that the TNM stage was higher than T3, N3, or M1; 10) Patients were sedated with additional dexmedetomidine for their condition after surgery. To obtain a detailed understanding of each BMI, especially groups that have not received much clinical attention, such as those who were overweight but not up to the standard of obesity and those who were lean but not up to cachexia, we grouped patients according to a certain gradient. They were placed into six groups: group A (BMI < $18.5 \mathrm{~kg} / \mathrm{m}^{2}, 29$ cases $)$, group B $\left(18.5 \mathrm{~kg} / \mathrm{m}^{2} \leq \mathrm{BMI}<22 \mathrm{~kg} / \mathrm{m}^{2}\right.$, 124 cases:), group C (22 kg/m² $\leq$ BMI $<24 \mathrm{~kg} / \mathrm{m}^{2}, 99$ cases $)$, and group $\mathrm{D}\left(24 \mathrm{~kg} / \mathrm{m}^{2} \leq \mathrm{BMI}<26 \mathrm{~kg} / \mathrm{m}^{2}, 69\right.$ cases $)$, group $\mathrm{E}$ $\left(26 \mathrm{~kg} / \mathrm{m}^{2} \leq \mathrm{BMI}<28 \mathrm{~kg} / \mathrm{m}^{2}, 46\right.$ cases $)$ and group $\mathrm{F}$ (BMI $\geq 28$ $\mathrm{kg} / \mathrm{m}^{2}, 23$ cases).

\section{Ethics}

The present study's protocols were approved by the Ethics Committee of West China Hospital, Sichuan University (Approval No. 306, 2018/9/5, Chairman: Professor Lunxu Liu). All procedures were in accordance with the ethical standards of the biomedical ethics committee of West China Hospital relevant Chinese laws and regulations on bioethics, as well as the ethical standards of the Helsinki Declaration and international ethical guidelines for human biomedical research.

\section{Analgesia Strategies}

None of the patients had a record of taking painkillers, including opioids, before surgery. All cases were treated with sufentanil during the operation. After the operation, the patients were treated with the same PCIA. The formula was sufentanil $2 \mu \mathrm{g} / \mathrm{kg}+$ flurbiprofen axetil $400 \mathrm{mg}+$ dexmedetomidine $200 \mu \mathrm{g}+$ methoxyclopramide hydrochloride $60 \mathrm{mg}+$ appropriate normal saline, a total of $200 \mathrm{~mL}$ analgesic solution. After the patient was connected to the automatic analgesia pump, the background 
dose was $2 \mathrm{~mL} / \mathrm{h}$, and the additional dose of automatic analgesia was $0.5 \mathrm{~mL} / 15 \mathrm{~min}$. Patients in severe pain were given dezocine for emergency analgesia.

\section{Data Collection}

1) Visual analogue score (VAS) and adverse reaction 24 hours (h) and $48 \mathrm{~h}$ after surgery; 2) Characteristics of patients, gender, age, height, and weight; 3) Preoperative complications, including hypertension and diabetes; 4) Intraoperative and postoperative tumor information; 5) Use of perioperative analgesics; 6) Time of operation; 7) The time from the end of the operation to the recovery of each activity, including passage of gas from anus, drinking water, getting out of bed, and removal of urine, gastric, and abdominal drainage tubes; 8) Postoperative hospital stay and living quality [Supplementary Material Page 2].

\section{Statistical Analysis}

All the above data were classified as quantitative or qualitative. One-way analysis of variance (one-way ANOVA) and $\chi^{2}$ tests were used to analyse the data. For those with positive results on one-way ANOVA, multiple comparisons were performed by Bonferroni. In addition, according to the data results, postoperative vomiting at $24 \mathrm{~h}$ was analysed by binary logistic regression analysis, expressed by odds ratio (OR) value and 95\% confidence interval (95\% CI). All data were entered into SPSS version 21.0 for statistical analysis.

\section{Results}

\section{Characteristics of Patients}

There were no significant differences in gender (Figure 1A$\mathrm{B}$ ), age (Figure 1C-E), height (Figure $1 \mathrm{~F}$ ), and tumor site (Figure 1H-I) between the groups $(p>0.05)$ (Table 1). Weight $(\mathrm{kg})$ of each subgroup was $45.79 \pm 4.31,54.73 \pm$ $5.66,61.05 \pm 5.93,65.46 \pm 6.89,70.77 \pm 7.08$, and $78.09 \pm$ 9.77, respectively (Figure $1 \mathrm{G}$ ). Each patient's BMI was calculated by dividing body weight by the square of height, so there was a significant difference in body weight between the groups $(\mathrm{p}=0<0.05)$ (Table 1$)$. After excluding other serious complications (heart disease, severe anaemia, etc.), patients with hypertension or diabetes were more common. From group A to group $\mathrm{F}$, the probability of hypertension was higher with increase of BMI $(p=0.012<0.05)$. There was little difference in incidence of diabetes mellitus among the subgroups $(p>0.05)$ (Table 1 and Figure $1 \mathrm{~J}-\mathrm{K})$.

\section{Duration of Operation and Use of Analgesics}

During the operation, there was no significant difference in the operative time among the subgroups $(p>0.05)$ (Table 2 and Figure 2A). Although one-way ANOVA showed significant differences in sufentanil dosage in each group $(p=0.01<0.05)$, further multiple comparisons showed no statistically significant differences between any two groups (Table 2 and Figure 2B). There was no difference between the use of other analgesics during operation (Figure 2C-D) and rescue analgesics after operation (Figure 2E-F) $(p>0.05)$ (Table 2).

\section{Postoperative VAS}

Regardless of the state of patients at each time point, postoperative VAS in groups $\mathrm{A}$ and $\mathrm{C}$ were higher than in other groups, especially when compared with group B (Table 3 and Figure 3A). Postoperative VAS of groups A and B were 1.83 \pm 1.56 and $1.10 \pm 0.98$, respectively, in the resting state of patients $24 \mathrm{~h}$ after surgery $(p=0.011<0.05)$. The mean VAS scores of each subgroup were $1.83 \pm 1.34,1.70 \pm 0.94,2.17 \pm$ $1.19,1.71 \pm 0.97,1.89 \pm 0.95$, and $1.78 \pm 0.80$, respectively, $48 \mathrm{~h}$ after the operation at movement. The difference between groups $\mathrm{B}$ and $\mathrm{C}$ was statistically significant $(p=0.013<0.05)$. A VAS greater than 3 indicates moderate to severe pain. Therefore, by comparing the proportion of patients with moderate to severe pain at each time point, the results show that the difference was meaningless (Table 3 and Figure 3B-C).

\section{Adverse Reactions}

There was no statistically significant difference in postoperative dizziness and nausea between groups A and $\mathrm{F}$ ( $\mathrm{p}$ $>0.05)$. It is worth noting that incidence of vomiting decreased gradually from group A to group F $24 \mathrm{~h}$ after surgery. The results were $10.3 \%$ (3/29), $3.2 \%$ (4/124), $2.0 \%$ (2/99), $1.4 \%(1 / 69), 0 \%(0 / 46)$ and $0 \%(0 / 23)$, respectively $(p=0.009<0.05)$ (Table 4 and Figure 4A-B). Binary logistic analysis found that the risk factors for vomiting $24 \mathrm{~h}$ after surgery were higher with age (OR: 1.158, 95\% CI: $1.003-1.377, p=0.045<0.05$ ) (Table 5).

\section{Postoperative Recovery}

There were no statistically significant differences in times to resume various activities after the operation, such as first anal exhaust (Figure 5A), drinking water (Figure 5B), getting out of bed (Figure 5C), removing the urination tube (Figure 5D), removing abdominal 


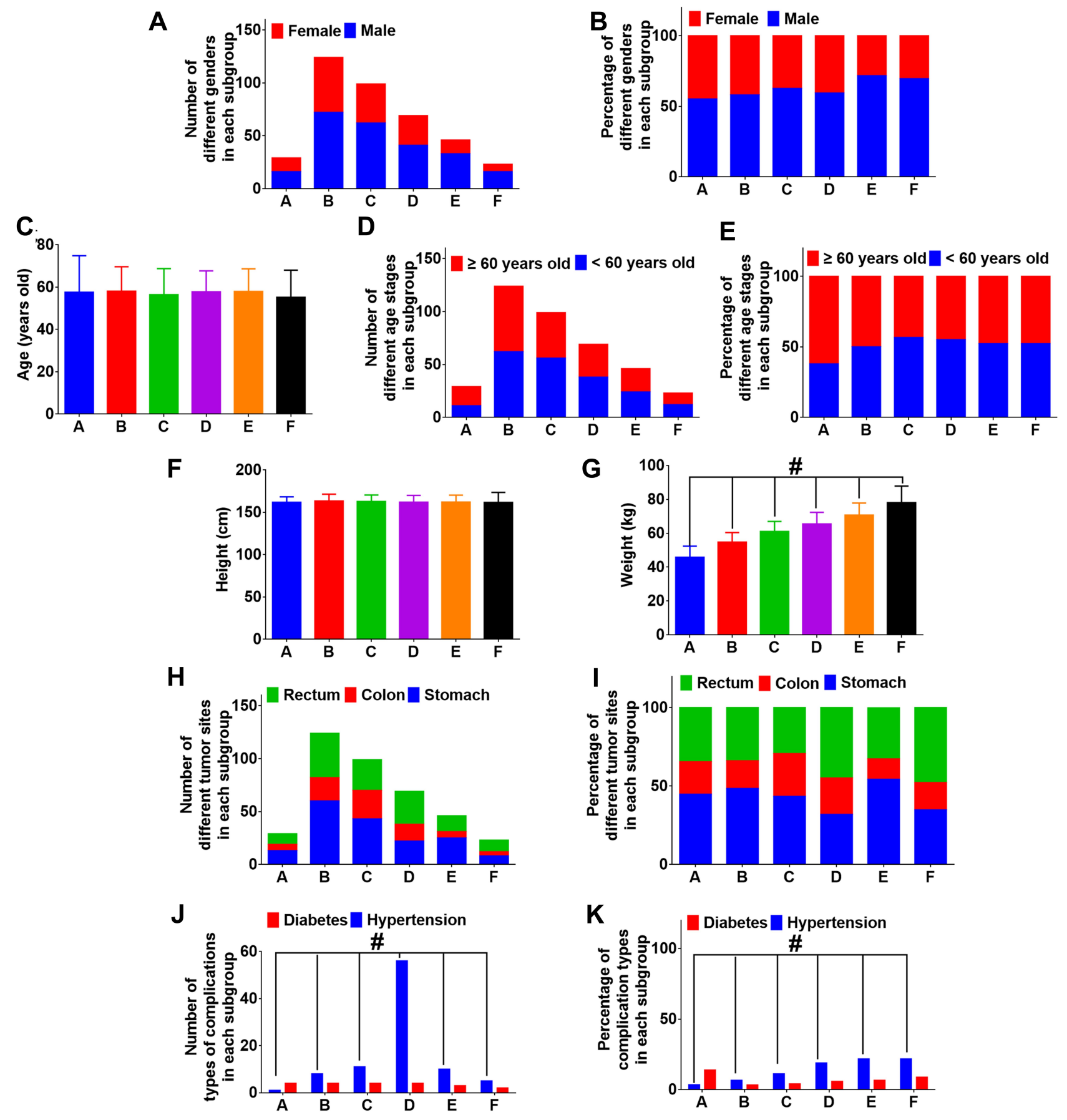

Figure I Characteristics of patients (A) Number of different genders in each subgroup. (B) Percentage of different genders in each subgroup. (C) Age of patients. (D) Number of different age stages in each subgroup. (E) Percentage of different age stages in each subgroup. (F) Height of patients. (G) Weight of patients. (H) Number of different tumor sites in each subgroup. (I) Percentage of different tumor sites in each subgroup. (J) Number of complications in each subgroup. (K) Percentage of complications in each subgroup. Notes: A: group A $\left(B M I<18.5 \mathrm{~kg} / \mathrm{m}^{2}\right) ;$ B: group B $\left(18.5 \mathrm{~kg} / \mathrm{m}^{2} \leq \mathrm{BMI}<22 \mathrm{~kg} / \mathrm{m}^{2}\right) ;$ C: group C $\left(22 \mathrm{~kg} / \mathrm{m}^{2} \leq \mathrm{BMI}<24 \mathrm{~kg} / \mathrm{m}^{2}\right) ;$ D: group D $\left(24 \mathrm{~kg} / \mathrm{m}^{2} \leq \mathrm{BMI}<26 \mathrm{~kg} / \mathrm{m}^{2}\right) ;$ E: group E $\left(26 \mathrm{~kg} / \mathrm{m}^{2} \leq \mathrm{BMI}<28 \mathrm{~kg} / \mathrm{m}^{2}\right) ; \mathrm{F}$ : group $\mathrm{F}\left(\mathrm{BMI} \geq 28 \mathrm{~kg} / \mathrm{m}^{2}\right)$. \#The difference of subgroups was statistically significant $(p<0.05)$.

drainage tube (Figure 5E), removing gastric tube (Figure 5F), and leaving the hospital (Figure 5G) $(p>$ 0.05 ) (Table 6). In addition, there were no significant differences in life quality assessments among the groups one month after surgery $(p>0.05)$ (Table 6 and Figure $5 \mathrm{H})$. 
Table I Characteristics of Patients

\begin{tabular}{|c|c|c|c|c|c|c|c|c|}
\hline \multirow{2}{*}{\multicolumn{2}{|c|}{ Group }} & $\mathbf{A}$ & B & C & D & $\mathbf{E}$ & $\mathbf{F}$ & \multirow{2}{*}{$\begin{array}{l}p \\
\text { value }\end{array}$} \\
\hline & & $\begin{array}{l}\text { BMI }<18.5 \\
\mathrm{~kg} / \mathrm{m}^{2}\end{array}$ & $\begin{array}{l}\mid 8.5 \mathrm{~kg} / \mathrm{m}^{2} \\
\leq \mathrm{BM} \mid<22 \mathrm{~kg} / \\
\mathrm{m}^{2}\end{array}$ & $\begin{array}{l}22 \mathrm{~kg} / \mathrm{m}^{2} \leq \\
\mathrm{BMI}<24 \mathrm{~kg} / \\
\mathrm{m}^{2}\end{array}$ & $\begin{array}{l}24 \mathrm{~kg} / \mathrm{m}^{2} \leq \\
\mathrm{BMI}<26 \mathrm{~kg} / \\
\mathrm{m}^{2}\end{array}$ & $\begin{array}{l}26 \mathrm{~kg} / \mathrm{m}^{2} \leq \\
\mathrm{BMI}<28 \mathrm{~kg} / \\
\mathrm{m}^{2}\end{array}$ & $\begin{array}{l}\text { BMI } \geq 28 \mathrm{~kg} / \\
\mathrm{m}^{2}\end{array}$ & \\
\hline \multirow[t]{2}{*}{ Gender (\%) } & Male & $55.2 \%(16 / 29)$ & $58.1 \%(72 / 124)$ & $62.6 \%(62 / 99)$ & $59.4 \%(4 I / 69)$ & $71.7 \%(33 / 46)$ & $69.6 \%(16 / 23)$ & 0.556 \\
\hline & Female & $44.8 \%$ (13/29) & $41.9 \%(52 / 124)$ & $37.4 \%$ (37/99) & $40.6 \%(28 / 69$ & $28.3 \%(13 / 46)$ & $30.4 \%(7 / 23)$ & \\
\hline \multirow[t]{3}{*}{ Age (years) } & Mean \pm SD & $57.55 \pm 17.30$ & $58.06 \pm 11.56$ & $56.42 \pm 12.35$ & $57.80 \pm 9.87$ & $57.96 \pm 10.70$ & $55.17 \pm 12.84$ & 0.88 \\
\hline & $<60$ & $37.9 \%(11 / 29)$ & $50.0 \%(62 / / 24)$ & $56.6 \%(56 / 99)$ & $55.1 \%(38 / 69)$ & $52.2 \%(24 / 46)$ & $52.2 \%(12 / 23)$ & 0.61 \\
\hline & $\geqq 60$ & $62.1 \%(18 / 29)$ & $50.0 \%(62 / 124)$ & $43.4 \%(43 / 99)$ & $44.9 \%(31 / 69)$ & $47.8 \%(22 / 46)$ & $47.8 \%(11 / 23)$ & \\
\hline Height (cm) & Mean \pm SD & $161.79 \pm 6.56$ & $163.40 \pm 8.08$ & $|62.75 \pm 7.7|$ & $162.01 \pm 8.06$ & $162.13 \pm 8.15$ & $161.70 \pm 11.94$ & 0.806 \\
\hline \multirow[t]{2}{*}{ Weight (kg) } & Mean \pm SD & $45.79 \pm 4.31$ & $54.73 \pm 5.66$ & $61.05 \pm 5.93$ & $65.46 \pm 6.89$ & $70.77 \pm 7.08$ & $78.09 \pm 9.77$ & $0 *$ \\
\hline & & $\begin{array}{l}\# b, \# c, \# d, \# e, \\
\# f\end{array}$ & $\begin{array}{l}\# \mathrm{a}, \# \mathrm{c}, \# \mathrm{~d}, \# \mathrm{e}, \\
\# \mathrm{f}\end{array}$ & $\begin{array}{l}\# \mathrm{a}, \# \mathrm{~b}, \# \mathrm{~d}, \\
\# \mathrm{e}, \# \mathrm{f}\end{array}$ & $\begin{array}{l}\# \mathrm{a}, \# \mathrm{~b}, \# \mathrm{c}, \\
\# \mathrm{e}, \# \mathrm{f}\end{array}$ & $\begin{array}{l}\# \mathrm{a}, \# \mathrm{~b}, \# \mathrm{c}, \\
\# \mathrm{~d}, \# \mathrm{f}\end{array}$ & $\begin{array}{l}\# \mathrm{a}, \# \mathrm{~b}, \# \mathrm{c}, \# \mathrm{~d}, \\
\# \mathrm{e}\end{array}$ & \\
\hline \multirow[t]{3}{*}{ Tumor location } & Stomach & $44.8 \%(13 / 29)$ & $48.4 \%(60 / / 24)$ & $43.4 \%(43 / 99)$ & $31.9 \%(22 / 69)$ & $54.3 \%(25 / 46)$ & $34.8 \%(8 / 23)$ & 0.244 \\
\hline & Colon & $20.7 \%(6 / 29)$ & $17.7 \%(22 / 124)$ & $27.3 \%$ (27/99) & $23.2 \%(16 / 69)$ & $13.0 \%(6 / 46)$ & 17.4\% (4/23) & \\
\hline & Rectum & $34.5 \%(10 / 29)$ & $33.9 \%(42 / 124)$ & $29.3 \%$ (29/99) & $44.9 \%(31 / 69)$ & $32.6 \%(15 / 46)$ & $47.8 \%(11 / 23)$ & \\
\hline \multicolumn{2}{|l|}{ Hypertension } & $3.4 \%(1 / 29)$ & $6.5 \%(8 / 116)$ & II.I\% (I I/99) & $18.8 \%(56 / 69)$ & $21.7 \%(10 / 46)$ & $21.7 \%(5 / 23)$ & $0.012 *$ \\
\hline \multicolumn{2}{|l|}{ Diabetes } & I3.8\% (4/29) & $3.2 \%(4 / 124)$ & $4.0 \%(4 / 99)$ & $5.8 \%(4 / 69)$ & $6.5 \%(3 / 46)$ & $8.7 \%(2 / 23)$ & 0.292 \\
\hline
\end{tabular}

Notes: A: BMI < $18.5 \mathrm{~kg} / \mathrm{m}^{2} ; \mathrm{B}: 18.5 \mathrm{~kg} / \mathrm{m}^{2} \leq \mathrm{BMI}<22 \mathrm{~kg} / \mathrm{m}^{2} ; \mathrm{C}: 22 \mathrm{~kg} / \mathrm{m}^{2} \leq \mathrm{BMI}<24 \mathrm{~kg} / \mathrm{m}^{2} ; \mathrm{D}: 24 \mathrm{~kg} / \mathrm{m}^{2} \leq \mathrm{BMI}<26 \mathrm{~kg} / \mathrm{m}^{2} ; \mathrm{E:}: 26 \mathrm{~kg} / \mathrm{m}^{2} \leq \mathrm{BMl}<28 \mathrm{~kg} / \mathrm{m}^{2} ; \mathrm{F}: \mathrm{BMI} \geq 28 \mathrm{~kg} / \mathrm{m}^{2}$. $* T h e r e$ were significant differences among groups $(p<0.05)$. \#a/\#b/\#c/\#d/\#e/\#f: The difference between this group and Group $A / B / C / D / E / F$ was statistically significant $(p<0.05)$. Abbreviation: SD, standard deviation.

Table 2 Duration of Operation and Use of Analgesics

\begin{tabular}{|c|c|c|c|c|c|c|c|c|c|}
\hline \multirow{2}{*}{\multicolumn{3}{|c|}{ Group }} & A & B & C & D & E & $\mathbf{F}$ & \multirow{2}{*}{$\begin{array}{l}P \\
\text { value }\end{array}$} \\
\hline & & & $\begin{array}{l}\mathrm{BMI}<18.5 \\
\mathrm{~kg} / \mathrm{m}^{2}\end{array}$ & $\begin{array}{l}18.5 \mathrm{~kg} / \mathrm{m}^{2} \\
\leq \mathrm{BMI}<22 \mathrm{~kg} / \\
\mathrm{m}^{2}\end{array}$ & $\begin{array}{l}22 \mathrm{~kg} / \mathrm{m}^{2} \leq \\
\mathrm{BMI}<24 \\
\mathrm{~kg} / \mathrm{m}^{2}\end{array}$ & $\begin{array}{l}24 \mathrm{~kg} / \mathrm{m}^{2} \leq \\
\mathrm{BMI}<26 \\
\mathrm{~kg} / \mathrm{m}^{2}\end{array}$ & $\begin{array}{l}26 \mathrm{~kg} / \mathrm{m}^{2} \leq \\
\mathrm{BMI}<28 \\
\mathrm{~kg} / \mathrm{m}^{2}\end{array}$ & $\begin{array}{l}\text { BMI } \geq 28 \\
\mathrm{~kg} / \mathrm{m}^{2}\end{array}$ & \\
\hline \multicolumn{3}{|c|}{ Time of operation (h) } & $2.98 \pm 0.99$ & $2.81 \pm 1.23$ & $2.95 \pm 1.22$ & $2.90 \pm 1.07$ & $3.25 \pm 1.37$ & $3.16 \pm 1.18$ & 0.364 \\
\hline \multirow[t]{3}{*}{ Analgesic } & $\begin{array}{l}\text { Intraoperative } \\
\text { dosage of sufentanil }\end{array}$ & Mean \pm SD & $38.90 \pm 14.36$ & $38.51 \pm 12.57$ & $39.52 \pm 13.43$ & $41.87 \pm 15.43$ & $46.48 \pm 18.94$ & $49.33 \pm 20.02$ & $0.01 *$ \\
\hline & $\begin{array}{l}\text { Use of other } \\
\text { analgesics during } \\
\text { operation (\%) }\end{array}$ & $\begin{array}{l}\text { Parecoxib } \\
\text { Flurbiprofen } \\
\text { axetil } \\
\text { Tramadol } \\
\text { Dezocine }\end{array}$ & $\begin{array}{l}37.9 \%(11 / 29) \\
58.6 \%(17 / 29) \\
0.0 \%(0 / 29) \\
3.4 \%(0 / 29)\end{array}$ & $\begin{array}{l}36.3 \%(45 / / 24) \\
48.4 \%(60 / / 24) \\
4.0 \%(5 / / 24) \\
12.1 \%(15 / / 24)\end{array}$ & $\begin{array}{l}36.4 \%(36 / 99) \\
49.5 \%(49 / 99) \\
3.0 \%(3 / 99) \\
12.1 \%(12 / 99)\end{array}$ & $\begin{array}{l}33.3 \%(23 / 69) \\
40.6 \%(28 / 69) \\
\\
5.8 \%(4 / 69) \\
20.3 \%(14 / 69)\end{array}$ & $\begin{array}{l}30.4 \%(14 / 46) \\
45.7 \%(21 / 46) \\
4.3 \%(2 / 46) \\
19.6 \%(9 / 46)\end{array}$ & $\begin{array}{l}47.8 \%(11 / 23) \\
43.5 \%(10 / 23) \\
0.0 \%(0 / 23) \\
8.7 \%(2 / 23)\end{array}$ & $\begin{array}{l}0.808 \\
0.673 \\
\\
0.678 \\
0.461\end{array}$ \\
\hline & $\begin{array}{l}\text { Use of additional } \\
\text { analgesics in the } \\
\text { postoperative ward } \\
\text { (\%) }\end{array}$ & $\begin{array}{l}\text { None } \\
\text { Sometimes } \\
\text { ( } \leq \text { twice) } \\
\text { Frequently } \\
\text { (> twice) }\end{array}$ & $\begin{array}{l}27.6 \%(8 / 29) \\
10.3 \%(3 / 29) \\
62.1 \%(18 / 29)\end{array}$ & $\begin{array}{l}29.8 \%(37 / 124) \\
12.9 \%(16 / 124) \\
57.3 \%(71 / 124)\end{array}$ & $\begin{array}{l}43.4 \%(43 / 99) \\
11.1 \%(11 / 99) \\
45.5 \%(45 / 99)\end{array}$ & $\begin{array}{l}42.0 \%(29 / 69) \\
10.1 \%(7 / 69) \\
47.8 \%(33 / 69)\end{array}$ & $\begin{array}{l}30.4 \%(14 / 46) \\
8.7 \%(4 / 46) \\
60.9 \%(28 / 46)\end{array}$ & $\begin{array}{l}26.1 \%(6 / 23) \\
13.0 \%(3 / 23) \\
60.9 \%(14 / 23)\end{array}$ & 0.539 \\
\hline
\end{tabular}

Notes: A: BMI < $18.5 \mathrm{~kg} / \mathrm{m} 2 ;$ B: $18.5 \mathrm{~kg} / \mathrm{m}^{2} \leq \mathrm{BMI}<22 \mathrm{~kg} / \mathrm{m}^{2} ; \mathrm{C}: 22 \mathrm{~kg} / \mathrm{m}^{2} \leq \mathrm{BMI}<24 \mathrm{~kg} / \mathrm{m}^{2} ;$ D: $24 \mathrm{~kg} / \mathrm{m}^{2} \leq \mathrm{BMl}<26 \mathrm{~kg} / \mathrm{m}^{2} ; \mathrm{E}: 26 \mathrm{~kg} / \mathrm{m}^{2} \leq \mathrm{BMI}<28 \mathrm{~kg} / \mathrm{m}^{2} ; \mathrm{F}: \mathrm{BMI} \geq$ $28 \mathrm{~kg} / \mathrm{m}^{2}$. *There were significant differences among groups $(p<0.05)$.

Abbreviation: SD, standard deviation.

\section{Discussion}

In this study, a total of 390 cases underwent the same

PCIA after open gastrointestinal surgery. Patients were placed into six groups according to BMI. Through oneway ANOVA, Bonferroni test and $\chi^{2}$ test, we found that, compared with patients with a BMI of $18.5-22 \mathrm{~kg} / \mathrm{m}^{2}$, 


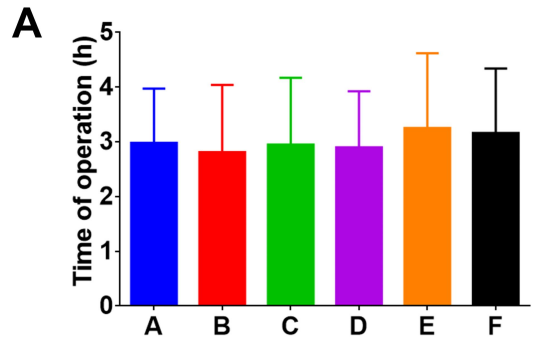

B

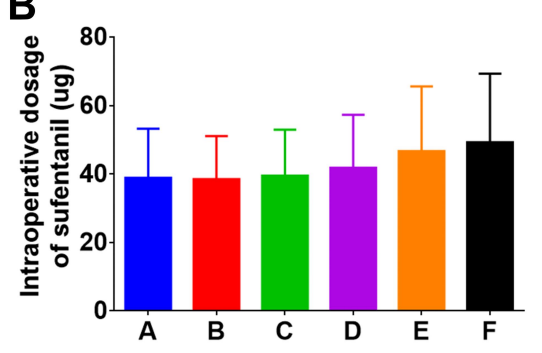

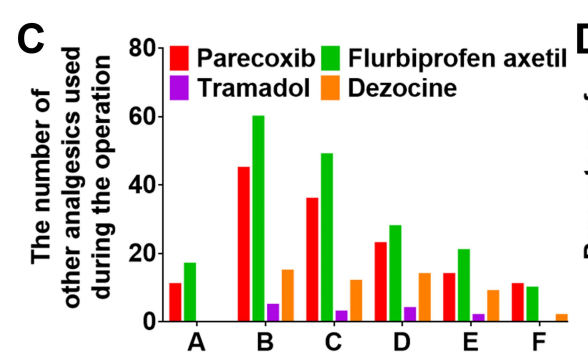

E

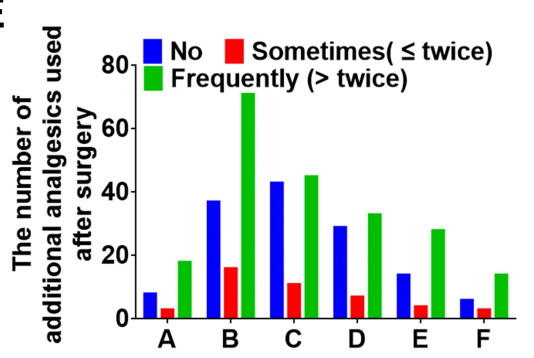

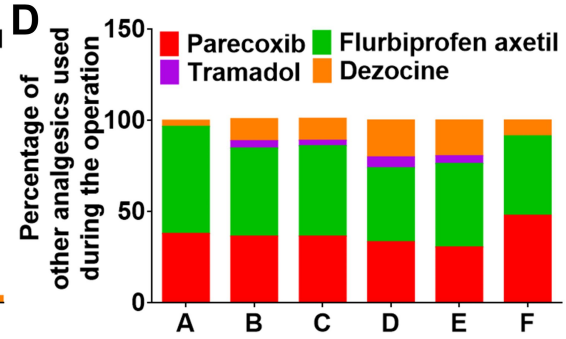

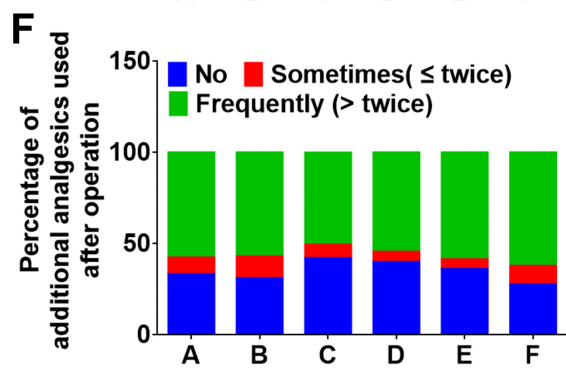

Figure 2 Duration of operation and use of analgesics. (A) Operation time of the patient (h). (B) Intraoperative use of sufentanil (ug). (C) The number of other analgesics used during the operation. (D) Percentage of other analgesics used during the operation. (E) The number of additional analgesics used after surgery. (F) Percentage of additional analgesics used after surgery.

Notes: A: group A $\left(B M I<18.5 \mathrm{~kg} / \mathrm{m}^{2}\right)$; B: group B $\left(18.5 \mathrm{~kg} / \mathrm{m}^{2} \leq\right.$ BMI $\left.<22 \mathrm{~kg} / \mathrm{m}^{2}\right)$; C: group C $\left(22 \mathrm{~kg} / \mathrm{m}^{2} \leq\right.$ BMI $\left.<24 \mathrm{~kg} / \mathrm{m}^{2}\right)$; D: group D $\left(24 \mathrm{~kg} / \mathrm{m}^{2} \leq\right.$ BMI < $\left.26 \mathrm{~kg} / \mathrm{m}^{2}\right)$; E: group E $\left(26 \mathrm{~kg} / \mathrm{m}^{2} \leq \mathrm{BMI}<28 \mathrm{~kg} / \mathrm{m}^{2}\right) ; \mathrm{F}$ : group $\mathrm{F}\left(B M I \geq 28 \mathrm{~kg} / \mathrm{m}^{2}\right)$.

individuals with a BMI of less than $18.5 \mathrm{~kg} / \mathrm{m}^{2}$ and of $22-24 \mathrm{~kg} / \mathrm{m}^{2}$ had higher postoperative VAS. Notably, compared with patients with BMI greater than $18.5 \mathrm{~kg} / \mathrm{m}^{2}$, those of less than $18.5 \mathrm{~kg} / \mathrm{m}^{2}$ had lower incidence of hypertension and higher incidence of vomiting $24 \mathrm{~h}$ after surgery. This suggests that we should pay more attention to the perioperative care of patients with BMIs of less than $18.5 \mathrm{~kg} / \mathrm{m}^{2}$.

\section{Postoperative Pain Assessment}

There were no significant differences in perioperative use of other analgesics and information on tumor diseases among the groups. Patients with BMI $\leq 18.5 \mathrm{~kg} / \mathrm{m}^{2}$ had a higher VAS than those of $18.5-22 \mathrm{~kg} / \mathrm{m}^{2}$ at rest on the first day after operation. Patients with a BMI of $22-24 \mathrm{~kg} / \mathrm{m}^{2}$ had a higher VAS than those of $18.5-22 \mathrm{~kg} / \mathrm{m}^{2}$ at movement on the second day after operation.

Table 3 VAS Score After Surgery

\begin{tabular}{|c|c|c|c|c|c|c|c|c|}
\hline \multirow{2}{*}{\multicolumn{2}{|c|}{ Group }} & A & B & C & D & E & $\mathbf{F}$ & \multirow{2}{*}{$\begin{array}{l}p \\
\text { value }\end{array}$} \\
\hline & & $\begin{array}{l}B M I<18.5 \\
\mathrm{~kg} / \mathrm{m}^{2}\end{array}$ & $\begin{array}{l}18.5 \mathrm{~kg} / \mathrm{m}^{2} \\
\leq B M \mid<22 \mathrm{~kg} / \mathrm{m}^{2}\end{array}$ & $\begin{array}{l}22 \mathrm{~kg} / \mathrm{m}^{2} \leq \text { BMI } \\
<24 \mathrm{~kg} / \mathrm{m}^{2}\end{array}$ & $\begin{array}{l}24 \mathrm{~kg} / \mathrm{m}^{2} \leq \mathrm{BMI} \\
<26 \mathrm{~kg} / \mathrm{m}^{2}\end{array}$ & $\begin{array}{l}26 \mathrm{~kg} / \mathrm{m}^{2} \leq \mathrm{BMI} \\
<28 \mathrm{~kg} / \mathrm{m}^{2}\end{array}$ & $\begin{array}{l}\text { BMI } \geq 28 \mathrm{~kg} / \\
\mathrm{m}^{2}\end{array}$ & \\
\hline $\begin{array}{l}\text { At rest } 24 \mathrm{~h} \\
\text { after surgery }\end{array}$ & $\begin{array}{l}\text { Mean } \pm S D \\
\text { VAS } \leq 3 \\
\text { VAS }>3\end{array}$ & $\begin{array}{l}1.83 \pm 1.56 \# b \\
93.1 \%(27 / 29) \\
6.9 \%(2 / 29)\end{array}$ & $\begin{array}{l}1.10 \pm 0.98 \# \mathrm{a} \\
97.6 \%(121 / 124) \\
2.4 \%(3 / 124)\end{array}$ & $\begin{array}{l}1.49 \pm 1.06 \\
96.0 \%(95 / 99) \\
4.0 \%(4 / 99)\end{array}$ & $\begin{array}{l}1.18 \pm 0.84 \\
100.0 \%(69 / 69) \\
0.0 \%(0 / 69)\end{array}$ & $\begin{array}{l}1.39 \pm 1.02 \\
97.8 \%(45 / 46) \\
2.2 \%(1 / 46)\end{array}$ & $\begin{array}{l}1.52 \pm 1.39 \\
100.0 \%(23 / 23) \\
0.0 \%(0 / 23)\end{array}$ & $\begin{array}{l}0.004^{*} \\
0.358\end{array}$ \\
\hline $\begin{array}{l}\text { Be active } 24 \mathrm{~h} \\
\text { after surgery }\end{array}$ & $\begin{array}{l}\text { Mean } \pm S D \\
\text { VAS } \leq 3 \\
\text { VAS }>3\end{array}$ & $\begin{array}{l}3.17 \pm 1.51 \\
62.1 \%(18 / 29) \\
37.9 \%(11 / 29)\end{array}$ & $\begin{array}{l}2.64 \pm 1.34 \\
78.2 \%(97 / 124) \\
21.8 \%(27 / 124)\end{array}$ & $\begin{array}{l}2.96 \pm 1.31 \\
67.7 \%(67 / 99) \\
32.3 \%(32 / 99)\end{array}$ & $\begin{array}{l}2.84 \pm 1.18 \\
73.9 \%(51 / 69) \\
26.1 \%(18 / 69)\end{array}$ & $\begin{array}{l}2.93 \pm 1.12 \\
73.9 \%(34 / 46) \\
26.1 \%(12 / 46)\end{array}$ & $\begin{array}{l}3.00 \pm 1.17 \\
65.3 \%(15 / 23) \\
34.8 \%(8 / 23)\end{array}$ & $\begin{array}{l}0.256 \\
0.361\end{array}$ \\
\hline $\begin{array}{l}\text { At rest } 48 \mathrm{~h} \\
\text { after surgery }\end{array}$ & $\begin{array}{l}\text { Mean } \pm S D \\
\text { VAS } \leq 3 \\
\text { VAS }>3\end{array}$ & $\begin{array}{l}0.72 \pm 1.10 \\
96.6 \%(28 / 29) \\
3.4 \%(1 / 29)\end{array}$ & $\begin{array}{l}0.56 \pm 0.76 \\
99.2 \%(123 / 124) \\
0.8 \%(1 / 124)\end{array}$ & $\begin{array}{l}0.60 \pm 0.77 \\
99.0 \%(98 / 99) \\
1.0 \%(1 / 99)\end{array}$ & $\begin{array}{l}0.48 \pm 0.63 \\
100.0 \%(69 / 69) \\
0.0 \%(0 / 69)\end{array}$ & $\begin{array}{l}0.59 \pm 0.72 \\
100.0 \%(46 / 46) \\
0.0 \%(0 / 46)\end{array}$ & $\begin{array}{l}0.61 \pm 0.72 \\
100.0 \%(23 / 23) \\
0.0 \%(0 / 23)\end{array}$ & $\begin{array}{l}0.796 \\
0.568\end{array}$ \\
\hline $\begin{array}{l}\text { Be active } 48 \mathrm{~h} \\
\text { after surgery }\end{array}$ & $\begin{array}{l}\text { Mean } \pm S D \\
\text { VAS } \leq 3 \\
\text { VAS }>3\end{array}$ & $\begin{array}{l}1.83 \pm 1.34 \\
96.6 \%(28 / 29) \\
3.4 \%(1 / 29)\end{array}$ & $\begin{array}{l}1.70 \pm 0.94 \# \mathrm{c} \\
96.0 \%(119 / 124) \\
4.0 \%(5 / 124)\end{array}$ & $\begin{array}{l}2.17 \pm 1.19 \# \mathrm{~b} \\
88.9 \%(88 / 99) \\
11.1 \%(11 / 99)\end{array}$ & $\begin{array}{l}I .71 \pm 0.97 \\
94.2 \%(65 / 69) \\
5.8 \%(4 / 69)\end{array}$ & $\begin{array}{l}1.89 \pm 0.95 \\
93.5 \%(43 / 46) \\
6.5 \%(3 / 46)\end{array}$ & $\begin{array}{l}1.78 \pm 0.80 \\
95.7 \%(22 / 23) \\
4.3 \%(1 / 23)\end{array}$ & $\begin{array}{l}0.021^{*} \\
0.364\end{array}$ \\
\hline
\end{tabular}

Notes: A: BMI < $18.5 \mathrm{~kg} / \mathrm{m}^{2} ; \mathrm{B}: 18.5 \mathrm{~kg} / \mathrm{m}^{2} \leq \mathrm{BMI}<22 \mathrm{~kg} / \mathrm{m}^{2} ; \mathrm{C}: 22 \mathrm{~kg} / \mathrm{m}^{2} \leq \mathrm{BMI}<24 \mathrm{~kg} / \mathrm{m}^{2} ; \mathrm{D}: 24 \mathrm{~kg} / \mathrm{m}^{2} \leq \mathrm{BMl}<26 \mathrm{~kg} / \mathrm{m}^{2} ; \mathrm{E}: 26 \mathrm{~kg} / \mathrm{m}^{2} \leq \mathrm{BMI}<28 \mathrm{~kg} / \mathrm{m}^{2} ; \mathrm{F}: \mathrm{BMI} \geq 28 \mathrm{~kg} /$ $\mathrm{m}^{2}$. *There were significant differences among groups $(p<0.05)$. \#a/\#b/\#c: The difference between this group and $\mathrm{Group} A / B / C$ was statistically significant $(p<0.05)$. Abbreviation: SD, standard deviation. 
Table 4 Postoperative Adverse Reactions

\begin{tabular}{|c|c|c|c|c|c|c|c|c|c|}
\hline \multirow{2}{*}{\multicolumn{3}{|c|}{ Group }} & $\mathbf{A}$ & B & C & D & $\mathbf{E}$ & $\mathbf{F}$ & \multirow{2}{*}{$\begin{array}{l}\boldsymbol{P} \\
\text { value }\end{array}$} \\
\hline & & & \multirow{2}{*}{$\begin{array}{l}\text { BMI < I8.5 } \\
\mathrm{kg} / \mathrm{m}^{2}\end{array}$} & $\begin{array}{l}18.5 \mathrm{~kg} / \mathrm{m}^{2} \\
\leq \mathrm{BM} \mid<22 \mathrm{~kg} / \\
\mathrm{m}^{2}\end{array}$ & $\begin{array}{l}22 \mathrm{~kg} / \mathrm{m}^{2} \leq \\
\text { BMI }<24 \mathrm{~kg} / \\
\mathrm{m}^{2}\end{array}$ & $\begin{array}{l}24 \mathrm{~kg} / \mathrm{m}^{2} \leq \\
\text { BMI }<26 \mathrm{~kg} / \\
\mathrm{m}^{2}\end{array}$ & $\begin{array}{l}26 \mathrm{~kg} / \mathrm{m}^{2} \leq \\
\text { BMI }<28 \mathrm{~kg} / \\
\mathrm{m}^{2}\end{array}$ & $\begin{array}{l}\text { BMI } \geq 28 \\
\mathrm{~kg} / \mathrm{m}^{2}\end{array}$ & \\
\hline \multirow{5}{*}{$\begin{array}{l}24 \mathrm{~h} \\
\text { after } \\
\text { surgery }\end{array}$} & \multirow[t]{3}{*}{ Dizzy } & Mild & & $8.3 \%(10 / 121)$ & $5.2 \%(5 / 97)$ & $10.1 \%(7 / 69)$ & $4.5 \%(2 / 44)$ & $0.0 \%(0 / 23)$ & \multirow[t]{3}{*}{0.554} \\
\hline & & Moderate & $0.0 \%(0 / 29)$ & $6.6 \%(8 / 121)$ & $4.1 \%(4 / 97)$ & $2.9 \%(2 / 69)$ & $6.8 \%(3 / 44)$ & $13.0 \%(3 / 23)$ & \\
\hline & & Severe & $3.4 \%(1 / 29)$ & $1.7 \%(2 /|2|)$ & $5.2 \%(5 / 97)$ & $1.4 \%(1 / 69)$ & $2.3 \%(I / 44)$ & $0.0 \%(0 / 23)$ & \\
\hline & \multicolumn{2}{|c|}{ Nausea } & $24.1 \%(7 / 29)$ & $10.5 \%(13 / 124)$ & $7.1 \%(7 / 99)$ & $8.7 \%(6 / 69)$ & $6.5 \%(3 / 46)$ & $8.7 \%(2 / 23)$ & 0.137 \\
\hline & \multicolumn{2}{|l|}{ Vomit } & $10.3 \%(3 / 29)$ & $3.2 \%(4 / 124)$ & $2.0 \%(2 / 99)$ & $1.4 \%(1 / 69)$ & $0.0 \%(0 / 46)$ & $0.0 \%(0 / 23)$ & $0.009 *$ \\
\hline \multirow{5}{*}{$\begin{array}{l}48 \mathrm{~h} \\
\text { after } \\
\text { surgery }\end{array}$} & \multirow[t]{3}{*}{ Dizzy } & Mild & $0.0 \%(0 / 29)$ & $0.0 \%(0 / 121)$ & $0.0 \%(0 / 98)$ & $0.0 \%(0 / 69)$ & $2.2 \%(1 / 45)$ & $4.3 \%(1 / 23)$ & \multirow[t]{3}{*}{0.23} \\
\hline & & Moderate & $0.0 \%(0 / 29)$ & $0.8 \%(|/| 2 \mid)$ & $0.0 \%(0 / 98)$ & $0.0 \%(0 / 69)$ & $0.0 \%(0 / 45)$ & $0.0 \%(0 / 23)$ & \\
\hline & & Severe & $0.0 \%(0 / 29)$ & $0.0 \%(0 / 121)$ & $0.0 \%(0 / 98)$ & $0.0 \%(0 / 69)$ & $0.0 \%(0 / 45)$ & $0.0 \%(0 / 23)$ & \\
\hline & \multicolumn{2}{|l|}{ Sick } & $3.4 \%(1 / 29)$ & $4.8 \%(6 / 124)$ & $4.0 \%(4 / 99)$ & $2.9 \%(2 / 69)$ & $0.0 \%(0 / 45)$ & $8.7 \%(2 / 23)$ & 0.582 \\
\hline & \multicolumn{2}{|l|}{ Vomit } & $0.0 \%(0 / 29)$ & $0.8 \%(\mathrm{I} / \mathrm{I} 24)$ & $2.0 \%(2 / 99)$ & $1.4 \%(1 / 69)$ & $0.0 \%(0 / 45)$ & $4.3 \%(\mathrm{I} / 23)$ & 0.65 \\
\hline
\end{tabular}

Notes: A: BMI < $18.5 \mathrm{~kg} / \mathrm{m}^{2} ; \mathrm{B}: 18.5 \mathrm{~kg} / \mathrm{m}^{2} \leq \mathrm{BMI}<22 \mathrm{~kg} / \mathrm{m}^{2} ;$ C: $22 \mathrm{~kg} / \mathrm{m}^{2} \leq \mathrm{BMI}<24 \mathrm{~kg} / \mathrm{m}^{2} ;$ D: $24 \mathrm{~kg} / \mathrm{m}^{2} \leq \mathrm{BMI}<26 \mathrm{~kg} / \mathrm{m}^{2} ; \mathrm{E}: 26 \mathrm{~kg} / \mathrm{m}^{2} \leq \mathrm{BMI}<28 \mathrm{~kg} / \mathrm{m}^{2} ; \mathrm{F}: \mathrm{BMI} \geq 28 \mathrm{~kg} /$ $\mathrm{m}^{2}$. $*$ There were significant differences among groups $(p<0.05)$.

Abbreviation: SD, standard deviation.

According to the above PCIA analgesic solution programme, the dose of sufentanil per unit weight was the same for all patients but dexmedetomidine and flurbiprofen were different. As described above, dexmedetomidine and flurbiprofen had the effect of enhancing analgesia. ${ }^{8-10,12}$ In theory, the new drug combination

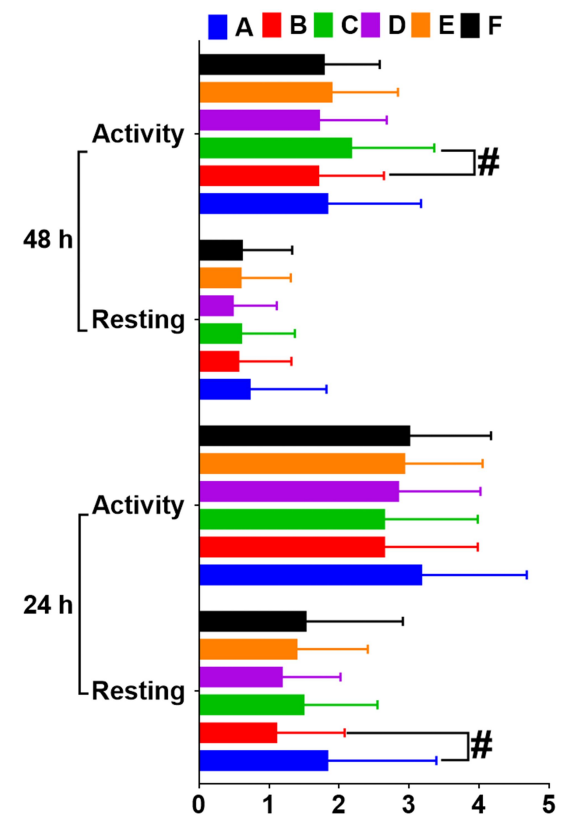

A Postoperative VAS
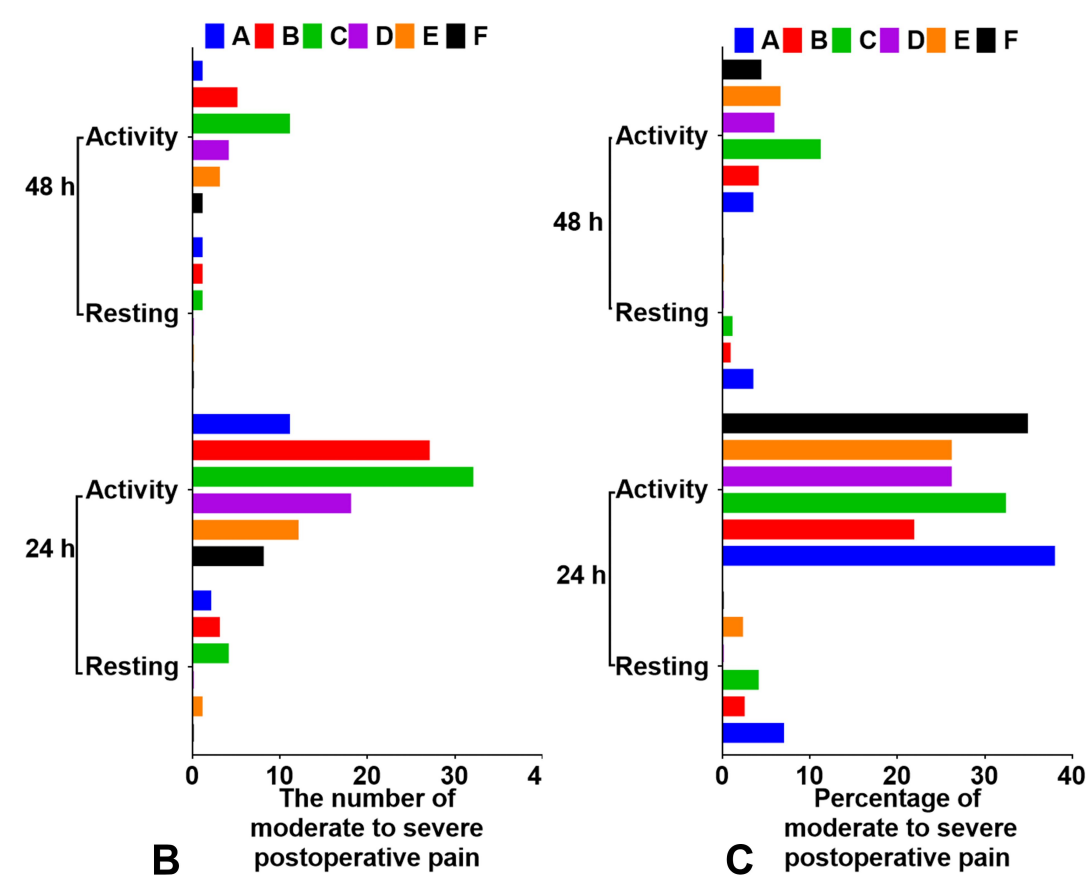

Figure 3 Postoperative VAS. (A) VAS scores at 24 hours and 48 hours after surgery. (B) The number of moderate to severe postoperative pain. (C) Percentage of moderate to severe postoperative pain.

Notes: A: group A $\left(\right.$ BMI < I8.5kg/m²); B: group B $\left(18.5 \mathrm{~kg} / \mathrm{m}^{2} \leq\right.$ BMI < $\left.22 \mathrm{~kg} / \mathrm{m}^{2}\right)$; C: group C $\left(22 \mathrm{~kg} / \mathrm{m}^{2} \leq \mathrm{BMI}<24 \mathrm{~kg} / \mathrm{m}^{2}\right)$; D: group D $\left(24 \mathrm{~kg} / \mathrm{m}^{2} \leq \mathrm{BMI}<26 \mathrm{~kg} / \mathrm{m}^{2}\right)$; E: group E $\left(26 \mathrm{~kg} / \mathrm{m}^{2} \leq \mathrm{BMI}<28 \mathrm{~kg} / \mathrm{m}^{2}\right) ; \mathrm{F}$ : group $\mathrm{F}\left(\mathrm{BMI} \geq 28 \mathrm{~kg} / \mathrm{m}^{2}\right)$. ${ }^{\text {\#T }}$ The difference of subgroups was statistically significant $(p<0.05)$. 
Table 5 Regression Analysis of Postoperative Vomiting

\begin{tabular}{|c|c|c|c|c|}
\hline & & OR & $95 \% \mathrm{Cl}$ & $p$ value \\
\hline Gender & $\begin{array}{l}\text { Male } \\
\text { Female }\end{array}$ & 0.526 & $0.526-5.371$ & 0.588 \\
\hline Age (years old) & Mean & 1.158 & $1.003-1.337$ & $0.045^{*}$ \\
\hline Height $(\mathrm{cm})$ & & 0.642 & $0.216-1.905$ & 0.424 \\
\hline Weight (kg) & & 1.875 & $0.382-9.215$ & 0.439 \\
\hline BMI (kg/m2) & & 0.119 & $0.002-8.689$ & 0.331 \\
\hline Tumor location & $\begin{array}{l}\text { Stomach } \\
\text { Colon } \\
\text { Rectum }\end{array}$ & 1.205 & $0.350-4.145$ & 0.767 \\
\hline Hypertension & & & & 0.997 \\
\hline Diabetes & & & & 0.998 \\
\hline Time of operation (h) & & 0.479 & $0.144-1.594$ & 0.230 \\
\hline Intraoperative sufentanil dosage (ug) & & 1.031 & $0.943-1.128$ & 0.498 \\
\hline Use of other analgesics during operation & $\begin{array}{l}\text { Parecoxib } \\
\text { Flurbiprofen axetil } \\
\text { Tramadol } \\
\text { Dezocine }\end{array}$ & & & $\begin{array}{l}1 \\
1 \\
0.998 \\
1\end{array}$ \\
\hline Postoperative VAS score & $\begin{array}{l}\text { At rest } 24 \mathrm{~h} \text { after surgery } \\
\text { Be active } 24 \mathrm{~h} \text { after surgery } \\
\text { At rest } 48 \mathrm{~h} \text { after surgery } \\
\text { Be active } 48 \mathrm{~h} \text { after surgery }\end{array}$ & $\begin{array}{l}4.284 \\
0.69 \\
0.103 \\
0.735\end{array}$ & $\begin{array}{l}0.623-29.481 \\
0.161-2.949 \\
0.01-1.039 \\
0.167-3.234\end{array}$ & $\begin{array}{l}0.139 \\
0.616 \\
0.054 \\
0.684\end{array}$ \\
\hline
\end{tabular}

Note: *The results were statistically significant $(p<0.05)$.

should be more effective in reducing pain in patients with low body weight because of the higher dose per unit of body weight of flurbiprofen and dexmedetomidine. Our results showed that these patients had a greater need for postoperative analgesics, which indicated that low BMI patients had a greater need for analgesics. Most current studies have focused on describing the effects of high BMI on analgesic effects, ${ }^{18,19}$ but little attention has been paid to patients with a low BMI $\left(\mathrm{BMI} \leq 18.5 \mathrm{~kg} / \mathrm{m}^{2}\right)$ or a normal high BMI $(22 \mathrm{~kg} / \mathrm{m} 2 \leq \mathrm{BMI}<24 \mathrm{~kg} / \mathrm{m} 2)$. Using logistic regression analysis, Lai et al found that a low BMI was an independent risk factor for colonoscopy pain. ${ }^{17}$ Trough a prospective longitudinal cohort study, Imagama $\mathrm{S}$ found that low BMI was an independent risk factor for neuropathic pain in middle-aged and elderly patients. ${ }^{20}$ In a study of 8- to 18-year-olds recovering from elective noncardiac surgery, Cohen B found that higher BMI was associated with a decrease in postoperative opioid consumption. ${ }^{19}$ Thus, patients with a BMI of less than
$18.5 \mathrm{~kg} / \mathrm{m}^{2}$ had a higher need for postoperative analgesia than patients with a BMI of $18.5-22 \mathrm{~kg} / \mathrm{m}^{2}$.

The results showed that patients with a BMI of less than $18.5 \mathrm{~kg} / \mathrm{m}^{2}$ had less hypertension than these with one greater than $18.5 \mathrm{~kg} / \mathrm{m}^{2}$. Studies show that people with higher hypertension rates have higher BMI levels. ${ }^{21}$ Using univariate analysis, Chiang et al showed that the BMIs of patients with hypertension were significantly higher than those with normal blood pressure, regardless of whether they were controlled with antihypertensive drugs. In addition, the study showed that antihypertensive drugs increase postoperative morphine use in women with hypertension, but not in men. ${ }^{22}$ The proportion of hypertension in BMI patients in each group were consistent with the above results. However, the effect of hypertension on pain in the above study may not be applicable to our results, as there were no separate comparisons between males and females, and no long-term preoperative medication of patients was obtained. At present, the main mechanism 

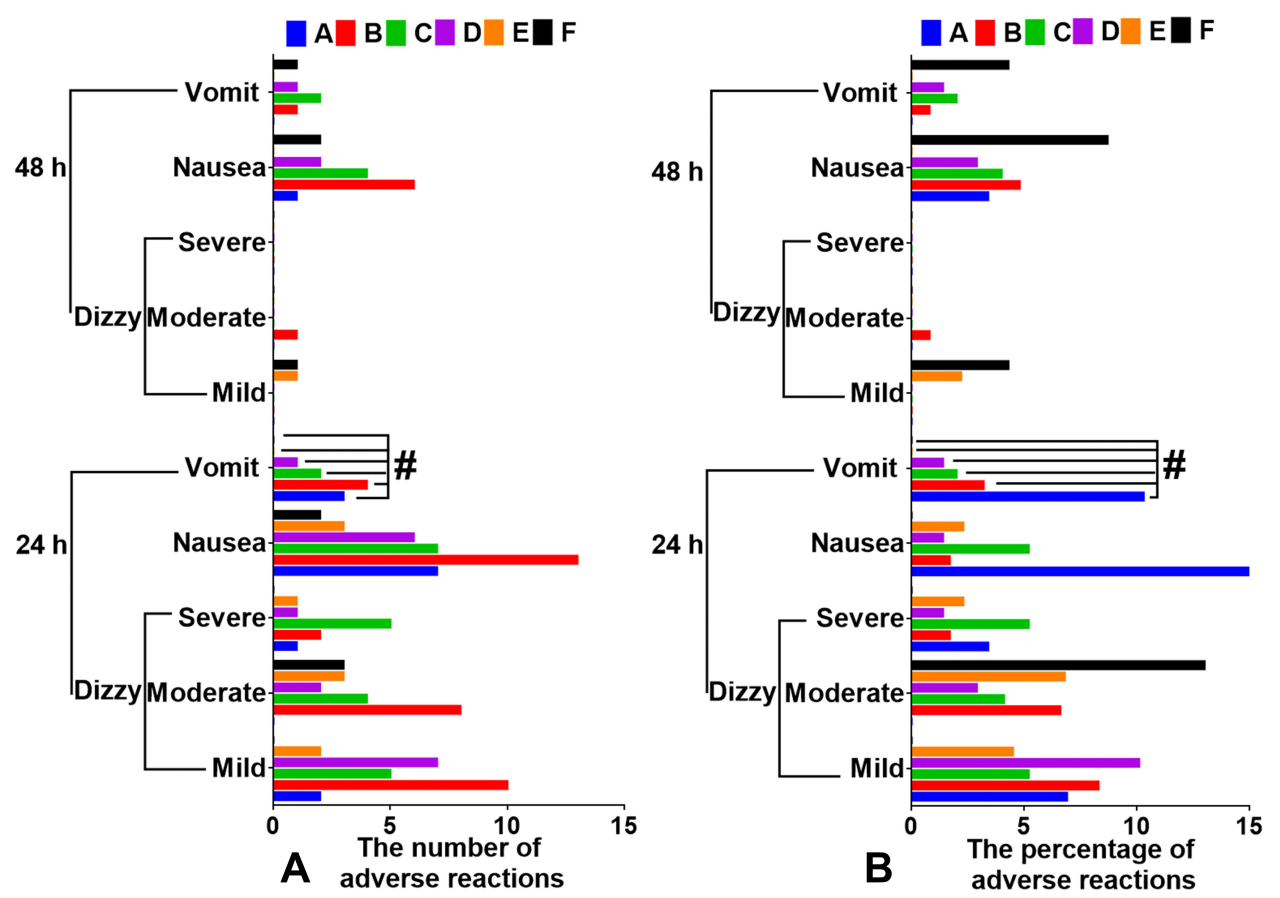

Figure 4 Postoperative adverse reactions. (A) The number of adverse reaction. (B) The percentage of adverse reaction.

Notes: A: group A $\left(B M I<18.5 \mathrm{~kg} / \mathrm{m}^{2}\right)$; B: group B $\left(18.5 \mathrm{~kg} / \mathrm{m}^{2} \leq \mathrm{BMI}<22 \mathrm{~kg} / \mathrm{m}^{2}\right) ;$ C: group C $\left(22 \mathrm{~kg} / \mathrm{m}^{2} \leq \mathrm{BMl}<24 \mathrm{~kg} / \mathrm{m}^{2}\right)$; D: group D $\left(24 \mathrm{~kg} / \mathrm{m}^{2} \leq \mathrm{BMI}<26 \mathrm{~kg} / \mathrm{m}^{2}\right) ; \mathrm{E}: \operatorname{group} E$ $\left(26 \mathrm{~kg} / \mathrm{m}^{2} \leq \mathrm{BMI}<28 \mathrm{~kg} / \mathrm{m}^{2}\right) ; \mathrm{F}$ : group $\mathrm{F}\left(\mathrm{BMI} \geq 28 \mathrm{~kg} / \mathrm{m}^{2}\right)$. ${ }^{\#}$ The difference of subgroups was statistically significant $(p<0.05)$.
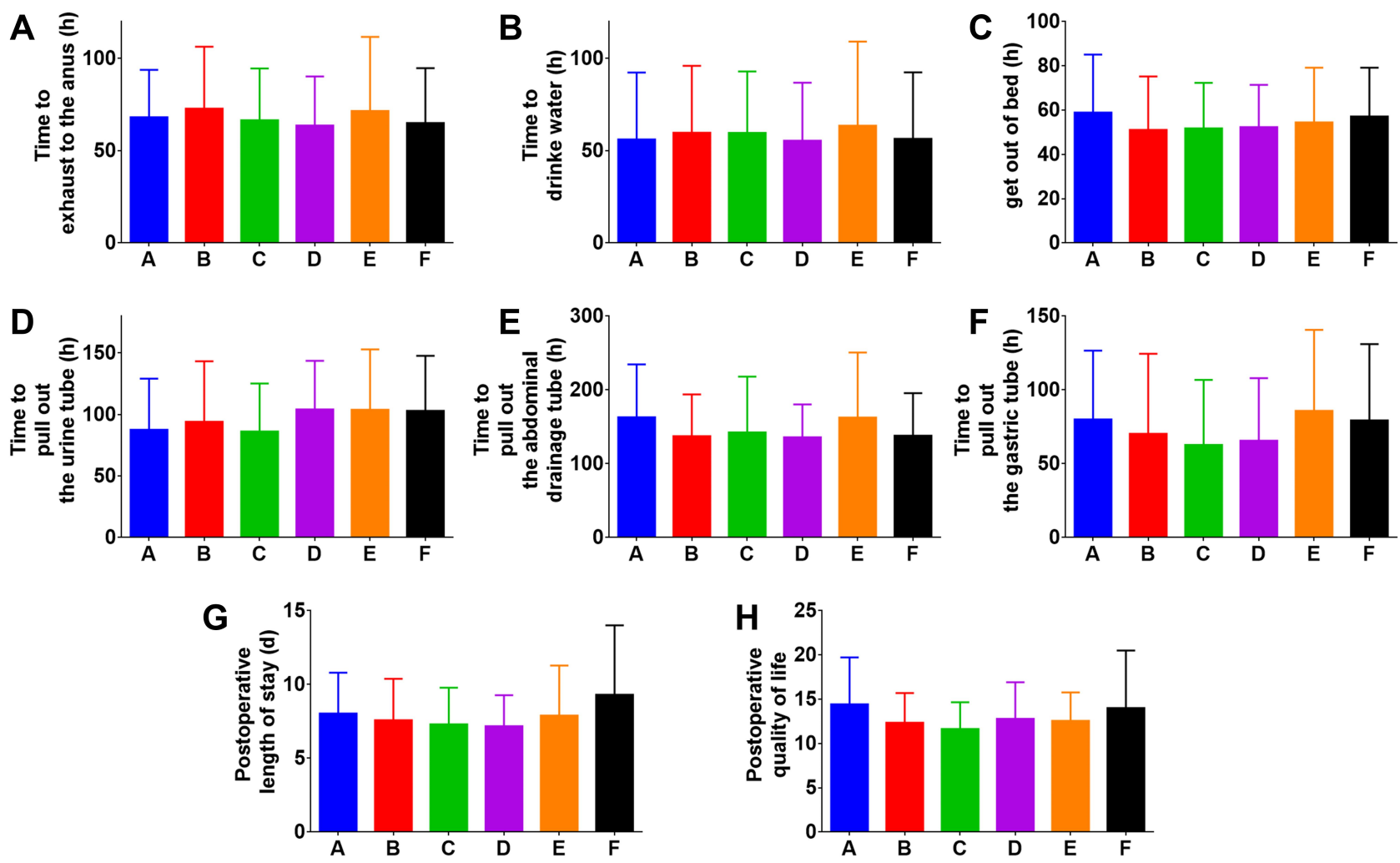

Figure 5 Time from the end of the operation to activities and postoperative quality of life. (A) Time to exhaust to the anus (h). (B) Time to drink water (h). (C) Time to get out of bed (h). (D) Time to pull out the urine tube (h). (E) Time to pull out the abdominal drainage tube (h). (F) Time to pull out the gastric tube (h). (G) Postoperative length of stay (d). (H) Postoperative quality of life.

Notes: A: group A (BMI < I8.5kg/m²); B: group B $\left(18.5 \mathrm{~kg} / \mathrm{m}^{2} \leq\right.$ BMI < $\left.22 \mathrm{~kg} / \mathrm{m}^{2}\right)$; C: group C $\left(22 \mathrm{~kg} / \mathrm{m}^{2} \leq \mathrm{BMI}<24 \mathrm{~kg} / \mathrm{m}^{2}\right)$; D: group D $\left(24 \mathrm{~kg} / \mathrm{m}^{2} \leq \mathrm{BMI}<26 \mathrm{~kg} / \mathrm{m}^{2}\right)$; E: group E $\left(26 \mathrm{~kg} / \mathrm{m}^{2} \leq \mathrm{BMI}<28 \mathrm{~kg} / \mathrm{m}^{2}\right)$; F: group $\mathrm{F}\left(\mathrm{BMI} \geq 28 \mathrm{~kg} / \mathrm{m}^{2}\right)$. 
Table 6 Postoperative Activity and Quality of Life Assessments

\begin{tabular}{|c|c|c|c|c|c|c|c|c|}
\hline \multirow{2}{*}{\multicolumn{2}{|c|}{ Group }} & $\mathbf{A}$ & B & C & D & E & $\mathbf{F}$ & \multirow{2}{*}{$\begin{array}{l}P \\
\text { value }\end{array}$} \\
\hline & & \multirow{2}{*}{$\begin{array}{l}\text { BMI }<18.5 \\
\mathrm{~kg} / \mathrm{m}^{2} \\
68.13 \pm 25.66\end{array}$} & \multirow{2}{*}{$\begin{array}{l}\begin{array}{l}18.5 \mathrm{~kg} / \mathrm{m}^{2} \\
\leq \mathrm{BMI}<22 \mathrm{~kg} / \\
\mathrm{m}^{2}\end{array} \\
72.8 \mathrm{I} \pm 33.48\end{array}$} & \multirow{2}{*}{$\begin{array}{l}22 \mathrm{~kg} / \mathrm{m}^{2} \\
\leq \mathrm{BMI}<24 \mathrm{~kg} / \\
\mathbf{m}^{2}\end{array}$} & \multirow{2}{*}{$\begin{array}{l}24 \mathrm{~kg} / \mathrm{m}^{2} \leq \\
\mathrm{BMI}<26 \mathrm{~kg} / \\
\mathrm{m}^{2}\end{array}$} & \multirow{2}{*}{$\begin{array}{l}26 \mathrm{~kg} / \mathrm{m}^{2} \leq \\
\mathrm{BMI}<28 \mathrm{~kg} / \\
\mathrm{m}^{2}\end{array}$} & \multirow{2}{*}{$\begin{array}{l}\text { BMI } \geq 28 \mathbf{~ k g} / \\
\mathbf{m}^{2} \\
64.98 \pm 29.76\end{array}$} & \\
\hline $\begin{array}{l}\text { The time from the } \\
\text { end of the }\end{array}$ & $\begin{array}{l}\text { Passage gas } \\
\text { anus (h) }\end{array}$ & & & & & & & 0.437 \\
\hline \multirow{5}{*}{ various activities } & Drinking (h) & $56.09 \pm 36.27$ & $59.77 \pm 36.27$ & $59.68 \pm 33.28$ & $55.45 \pm 31.42$ & $63.55 \pm 45.63$ & $56.45 \pm 35.94$ & 0.88 \\
\hline & $\begin{array}{l}\text { Getting out } \\
\text { of bed(h) }\end{array}$ & $58.89 \pm 26.20$ & $51.03 \pm 24.14$ & $51.76 \pm 20.56$ & $52.31 \pm 19.08$ & $54.46 \pm 24.67$ & $57.09 \pm 22.06$ & 0.532 \\
\hline & $\begin{array}{l}\text { Pulling out } \\
\text { the urine } \\
\text { tube(h) }\end{array}$ & $87.51 \pm 41.61$ & $94.04 \pm 49.28$ & $86.22 \pm 39.05$ & $104.18 \pm 39.44$ & $103.80 \pm 49.07$ & $102.93 \pm 44.79$ & 0.094 \\
\hline & $\begin{array}{l}\text { Pulling out } \\
\text { the } \\
\text { abdominal } \\
\text { drainage } \\
\text { tube (h) }\end{array}$ & $163.03 \pm 71.43$ & $137.34 \pm 56.57$ & $142.33 \pm 75.53$ & $135.86 \pm 44.30$ & $162.52 \pm 88.02$ & $137.96 \pm 57.67$ & 0.207 \\
\hline & $\begin{array}{l}\text { Pulling out } \\
\text { the gastric } \\
\text { tube(h) }\end{array}$ & $79.96 \pm 46.59$ & $70.17 \pm 54.31$ & $62.73 \pm 44.06$ & $65.62 \pm 42.35$ & $85.79 \pm 54.94$ & $79.4 I \pm 51.55$ & 0.48 \\
\hline \multicolumn{2}{|c|}{ Postoperative length of stay(d) } & $8.03 \pm 2.77$ & $7.57 \pm 2.81$ & $7.30 \pm 2.48$ & $7.17 \pm 2.10$ & $7.89 \pm 3.39$ & $9.30 \pm 4.70$ & 0.101 \\
\hline \multicolumn{2}{|c|}{ Postoperative quality of life } & $14.43 \pm 5.28$ & $12.33 \pm 3.37$ & $11.63 \pm 3.02$ & $12.78 \pm 4.13$ & $12.56 \pm 3.20$ & $14.00 \pm 6.49$ & 0.146 \\
\hline
\end{tabular}

Notes: A: BMI < $18.5 \mathrm{~kg} / \mathrm{m}^{2} ; \mathrm{B}: 18.5 \mathrm{~kg} / \mathrm{m}^{2} \leq \mathrm{BMI}<22 \mathrm{~kg} / \mathrm{m}^{2} ; \mathrm{C}: 22 \mathrm{~kg} / \mathrm{m}^{2} \leq \mathrm{BMl}<24 \mathrm{~kg} / \mathrm{m}^{2} ;$ D: $24 \mathrm{~kg} / \mathrm{m}^{2} \leq \mathrm{BMl}<26 \mathrm{~kg} / \mathrm{m}^{2} ; \mathrm{E}: 26 \mathrm{~kg} / \mathrm{m}^{2} \leq \mathrm{BMl}<28 \mathrm{~kg} / \mathrm{m}^{2} ; \mathrm{F}: \mathrm{BMl} \geq 28 \mathrm{~kg} / \mathrm{m}^{2}$.

of pain sensitivity caused by hypertension is impaired cardiovascular regulation. ${ }^{23}$ Considering that BMI can be used to assess body's nutritional status, ${ }^{24,25}$ we speculate that BMI effects on pain may be related to impaired vascular regulation. But this needs further research. In this study, compared with patients with a BMI of 18.5$22 \mathrm{~kg} / \mathrm{m}^{2}$, those with less than $18.5 \mathrm{~kg} / \mathrm{m}^{2}$ and those with $22-24 \mathrm{~kg} / \mathrm{m}^{2}$ had relatively higher postoperative VAS, especially when resting for $24 \mathrm{~h}$ and at movement for 48 h. This suggests that we need to increase postoperative attention to this group.

\section{Postoperative Adverse Reactions}

Compared with patients with a BMI of more than $18.5 \mathrm{~kg} /$ $\mathrm{m}^{2}$, those of less than $18.5 \mathrm{~kg} / \mathrm{m}^{2}$ had a significantly higher probability of vomiting $24 \mathrm{~h}$ after surgery. Through multifactor analysis, some study showed that patients with lower BMI were more likely to have postoperative complications such as nausea and vomiting. ${ }^{26}$ Similar studies have shown that patients with low BMI are more likely to develop PONV. $^{27-29}$ These are consistent with our results in part. Notably, some studies have shown that dexmedetomidine for postoperative analgesia can reduce the occurrence of PONV. ${ }^{30,31}$ Through mate analysis, Sun et al confirmed that perioperative flurbiprofen can effectively reduce PONV in Chinese surgical patients. ${ }^{13}$ Metoclopramide produces antiemetic effects by acting on dopamine or serotonin, and has long been used for postoperative vomiting. ${ }^{32}$ Combination of these drugs reduced the incidence of opioid-induced postoperative vomiting, which explained the small difference in the probability of PONV among the subgroups at most time points. PONV in patients with low BMI has been widely reported, but it is not clear why PONV is higher in cases of low BMI. In a study of the relationship between vomiting during pregnancy and low BMI, Ben-Aroya $\mathrm{Z}$ pointed out that hyperventilation affected vestibular dependence in women with low BMI, leading to more frequent vomiting in the first trimester. ${ }^{33}$ Postoperative analgesia was worse in patients with low BMI on the first day after surgery, and their pain may cause them to hyperventilate increasing the probability of vomiting.

In summary, in the case of sufentanil combined with dexmedetomidine and flurbiprofen for PCIA after open gastrointestinal tumor surgery, patients with a BMI of less than 
$18.5 \mathrm{~kg} / \mathrm{m}^{2}$ had the worst pain relief on the first day after surgery, compared with patients with a BMI of more than $18.5 \mathrm{~kg} / \mathrm{m}^{2}$, and especially those with a BMI of 18.5 to $22 \mathrm{~kg} /$ $\mathrm{m}^{2}$. In addition, patients with a BMI of less than $18.5 \mathrm{~kg} / \mathrm{m}^{2}$ had less hypertension and were more likely to experience postoperative vomiting on the first day after surgery than individuals with a greater BMI. This study retrospectively compares the analgesic effects and related adverse reactions of patients with different BMIs under the same PCIA, which fills a gap in studies of the correlation between different BMIs and PCIAs. However, there are too few cases of BMIs of less than $18.5 \mathrm{~kg} / \mathrm{m}^{2}$ and higher than $28 \mathrm{~kg} / \mathrm{m}^{2}$ in this paper. In addition, this result is based only on the analgesic method we used, which cannot be applied to other PCIAs.

\section{Conclusions}

The combination of dexmedetomidine, sufentanil and flurbiprofen axetil was used for PCIA after open gastrointestinal tumor surgery. Compared to those with a BMI greater than $18.5 \mathrm{~kg} / \mathrm{m}^{2}$, patients with a BMI of less than $18.5 \mathrm{~kg} /$ $\mathrm{m}^{2}$ had poorer analgesic effects on the first day after surgery and were more prone to vomiting. Postoperative analgesia and related experiences in patients with BMIs of less than $18.5 \mathrm{~kg} / \mathrm{m}^{2}$ need to be improved.

\section{Disclosure}

It should be understood that none of the authors have any financial or scientific conflicts of interest with regard to the research described in this manuscript. The authors report no conflicts of interest for this work.

\section{References}

1. Zku Z, Wang C, Xu C, Cai Q. Influence of patient-controlled epidural analgesia versus patient-controlled intravenous analgesia on postoperative pain control and recovery after gastrectomy for gastric cancer: a prospective randomized trial. Gastric Cancer. 2013;16(2):193-200. doi:10.1007/s10120-012-0168-Z

2. Wang L, Li X, Cheng H, Liang J, Wang Y. Effect of patient-controlled epidural analgesia versus patient-controlled intravenous analgesia on postoperative pain management and short-term outcomes after gastric cancer resection: a retrospective analysis of 3042 consecutive patients between 2010 and 2015. J Pain Res. 2018;11:1743-1749.

3. Brodner G, Mertes N, Buerkle H, Marcus MA, Van Aken H. Acute pain management: analysis, implications and consequences after prospective experience with 6349 surgical patients. Eur J Anaesthesiol. 2000;17(9):566-575. doi:10.1046/j.1365-2346.2000.00738.x

4. K S, K S, N M, et al. Comparison of the effects of epidural analgesia and patient-controlled intravenous analgesia on postoperative pain relief and recovery after laparoscopic gastrectomy for gastric cancer. Surg Laparosc Endosc Percutan Tech. 2019;29(5):405-408. doi:10.1097/SLE.000000000000605
5. Mohamad M, Mohammad M, Hetta D, Ahmed E, Obiedallah A, Elzohry A. Thoracic epidural analgesia reduces myocardial injury in ischemic patients undergoing major abdominal cancer surgery. $J$ Pain Res. 2017;10:887-895. doi:10.2147/JPR.S122918

6. Rosow C. Sufentanil citrate: a new opioid analgesic for use in anesthesia. Pharmacotherapy. 1984;4(1):11-19. doi:10.1002/j.18759114.1984.tb03304.x

7. Apfelbaum J, Chen C, Mehta S, Gan T. Postoperative pain experience: results from a national survey suggest postoperative pain continues to be undermanaged. Anesth Analg. 2003;97(2):534-540, table of contents. doi:10.1213/01.ANE.0000068822.10113.9E

8. Afonso J, Reis F. Dexmedetomidine: current role in anesthesia and intensive care. Rev Bras Anestesiol. 2012;62(1):118-133. doi:10.1016/S0034-7094(12)70110-1

9. Qin M, Chen K, Liu T, Shen X. Dexmedetomidine in combination with sufentanil for postoperative analgesia after partial laryngectomy. BMC Anesthesiol. 2017;17(1):66. doi:10.1186/s12871-017-0363-x

10. Xin J, Zhang Y, Zhou L, et al. Effect of dexmedetomidine infusion for intravenous patient-controlled analgesia on the quality of recovery after laparotomy surgery. Oncotarget. 2017;8(59):100371-100383. doi:10.18632/oncotarget.22232

11. Piao G, Wu J. Systematic assessment of dexmedetomidine as an anesthetic agent: a meta-analysis of randomized controlled trials. Arch Med Sci. 2014;10(1):19-24. doi:10.5114/aoms.2014.40730

12. Martinez L, Ekman E, Nakhla N. Perioperative opioid-sparing strategies: utility of conventional NSAIDs in adults. Clin Ther. 2019;41 (12):2612-2628. doi:10.1016/j.clinthera.2019.10.002

13. Sun M, Cong X, Chang E, Miao M, Zhang J. Efficacy of flurbiprofen for postoperative pain in chinese surgical patients: a meta-analysis. $J$ Surg Res. 2020;252:80-88. doi:10.1016/j.jss.2019.11.032

14. Yanjun-Chen L-X, Li T. Analgesic Effect of Sufentanil Combined with Dexmedetomidine and Flurbiprofen Axetil after Gastrointestinal Surgery: A Retrospective Analysis. 2020.

15. Majchrzak M, Daroszewski C, Błasiak P, et al. Increased pain sensitivity in obese patients after lung cancer surgery. Front Pharmacol. 2019;10:626. doi:10.3389/fphar.2019.00626

16. Tashani OA, Astita R, Sharp D, Johnson MI. Body mass index and distribution of body fat can influence sensory detection and pain sensitivity. Eur J Pain. 2017;21(7):1186-1196. doi:10.1002/ejp.1019

17. Lai X-Y, Tang X-W, Huang S-L, et al. [Risk factors of pain during colonoscopic examination]. Nan Fang Yi Ke Da Xue Xue Bao = Journal of Southern Medical University. 2016;37(4):482-487. Chinese.

18. Patanwala AE, Holmes KL, Erstad BL. Analgesic response to morphine in obese and morbidly obese patients in the emergency department. Emerg Med J. 2014;31(2):139-142. doi:10.1136/emermed2012-202168

19. C B, Tanios MA, K O, et al. Association between higher BMI and postoperative pain and opioid consumption in pediatric inpatients - a retrospective cohort study. J Clin Anesth. 2020;62:109729. doi:10.1016/j.jclinane.2020.109729

20. Imagama S, Ando K, Kobayashi K, et al. Risk factors for neuropathic pain in middle-aged and elderly people: a five-year longitudinal cohort in the Yakumo Study. Pain Med. 2020;21(8):1604-1610. doi:10.1093/pm/pnaa036

21. Giummarra M, Tardif H, Blanchard M, Tonkin A, Arnold C. Hypertension prevalence in patients attending tertiary pain management services, a registry-based Australian cohort study. PLoS One. 2020;15(1):e0228173. doi:10.1371/journal.pone.0228173

22. Chiang H, Huang Y, Lin H, Chan M, Chia Y. Hypertension and postoperative pain: a prospective observational study. Pain Res Manage. 2019;2019:8946195. doi:10.1155/2019/8946195

23. Bruehl S, Olsen R, Tronstad C, et al. Chronic pain-related changes in cardiovascular regulation and impact on comorbid hypertension in a general population: the Tromsø study. Pain. 2018;159(1):119-127. doi:10.1097/j.pain.0000000000001070 
24. Marini Ana CB, Pimentel GD. Is body weight or muscle strength correlated with the Malnutrition Inflammation Score (MIS)? A crosssectional study in hemodialysis patients. Clin Nutri ESPEN. 2019;33:276-278. doi:10.1016/j.clnesp.2019.07.009

25. Liang X, Shu W, Linyong Z, et al. Preoperative nutritional evaluation of patients with hepatic alveolar echinococcosis. PLoS One. 2020;15 (2):e0229396. doi:10.1371/journal.pone.0229396

26. Schürner AM, Manzini G, Bueter M, Schadde E, Beck-Schimmer B, Schläpfer M. Perioperative surgery- and anaesthesia-related risks of laparoscopic Roux-en-Y gastric bypass - a single centre, retrospective data analysis. BMC Anesthesiol. 2018;18(1):190. doi:10.1186/s12871018-0654-X

27. N K, S Y, S S, H T, H K. Risk factors for nausea and vomiting following vitrectomyin adults. Eur J Anaesthesiol. 2007;24(2):166170. doi:10.1017/S0265021506001360

28. O M, Y H. [Comparison of analgesics used during balanced anesthesia on the incidence of PONV]. Masui Japanese J Anesthesiol. 2004;53(2):161-166. Japanese.

29. Ukai T, Ebihara G, Watanabe M. Opioid administration via epidural catheter is a risk factor for postoperative nausea and vomiting in total hip arthroplasty: a retrospective study. J Orthopaedic Sci. 2018;23 (6):973-976. doi:10.1016/j.jos.2018.06.011
30. Naduvanahalli Vivekanandaswamy A, Prasad Shetty A, Mugesh Kanna R, Shanmuganathan R. An analysis of the safety and efficacy of dexmedetomidine in posterior spinal fusion surgery for adolescent idiopathic scoliosis: a prospective randomized study. Eur Spine J. 2020. doi:10.1007/s00586-020-06539-9

31. Reddy S, Jones J, Gordish-Dressman H, Pestieau S. Dexmedetomidine as an opioid-sparing agent in pediatric craniofacial surgery. Children (Basel, Switzerland). 2020;7:7.

32. Dulay M, Dulay J. Antiemetics: types, actions and uses. Br J Hosp Med. 2020;81(5):1-8. doi:10.12968/hmed.2020.0050

33. Ben-Aroya Z, Lurie S, Segal D, Hallak M, Glezerman M. Association of nausea and vomiting in pregnancy with lower body mass index. Eur J Obstet Gynecol Reprod Biol. 2005;118(2):196198. doi:10.1016/j.ejogrb.2004.04.026

\section{Publish your work in this journal}

The Journal of Pain Research is an international, peer reviewed, open access, online journal that welcomes laboratory and clinical findings in the fields of pain research and the prevention and management of pain. Original research, reviews, symposium reports, hypothesis formation and commentaries are all considered for publication. The manuscript management system is completely online and includes a very quick and fair peer-review system, which is all easy to use. Visit http:// www.dovepress.com/testimonials.php to read real quotes from published authors. 